\title{
Ultrafast Third Harmonic Micro-spectroscopy Reveals a Two-Photon Resonance in Human Hemoglobin
}

\author{
G. Omar Clay ${ }^{1}$, Chris B. Schaffer ${ }^{1}$, Jeffrey A. Squier ${ }^{2}$ and David Kleinfeld ${ }^{1}$ \\ ${ }^{1}$ Department of Physics \\ University of California at San Diego \\ La Jolla CA 92093 \\ ${ }^{2}$ Department of Physics \\ Colorado School of Mines \\ Golden, CO 80401
}

\begin{abstract}
The recently developed technique of ultrafast third harmonic generation (THG) micro-spectroscopy is discussed. The approach is easily adapted to a standard laser scanning microscope and allows for two and three photon resonances to be identified in non-fluorescent unlabeled samples. This work provides nonlinear microscopists with a tool for further understanding the contrast and damage mechanisms they will encounter under nonlinear excitation. Here, we use THG micro-spectroscopy to investigate the nonlinear optical properties of hemoglobin over the spectral range of $770-1000$ $\mathrm{nm}$ with $100 \mathrm{fs}$ long $\mathrm{nJ}$ energy laser pulses. We demonstrate the ability of this approach to distinguish different ligand binding states in physiological solutions of human hemoglobin.
\end{abstract}

Keywords: $\quad$ Third Harmonic Generation, Micro-spectroscopy, Nonlinear Spectroscopy, Two-photon Microscopy, Hemoglobin, Two-photon Absorption, Refractive Index, Multi-photon Absorption, Third Harmonic Microscopy, Nonlinear Resonance

$\begin{array}{lll}\text { Abbreviations: } & \text { Ultraviolet } & (\mathrm{UV}) \\ & \text { Silicon Dioxide } & \left(\mathrm{SiO}_{2}\right) \\ & \text { Third Harmonic Generation } & (\mathrm{THG})\end{array}$

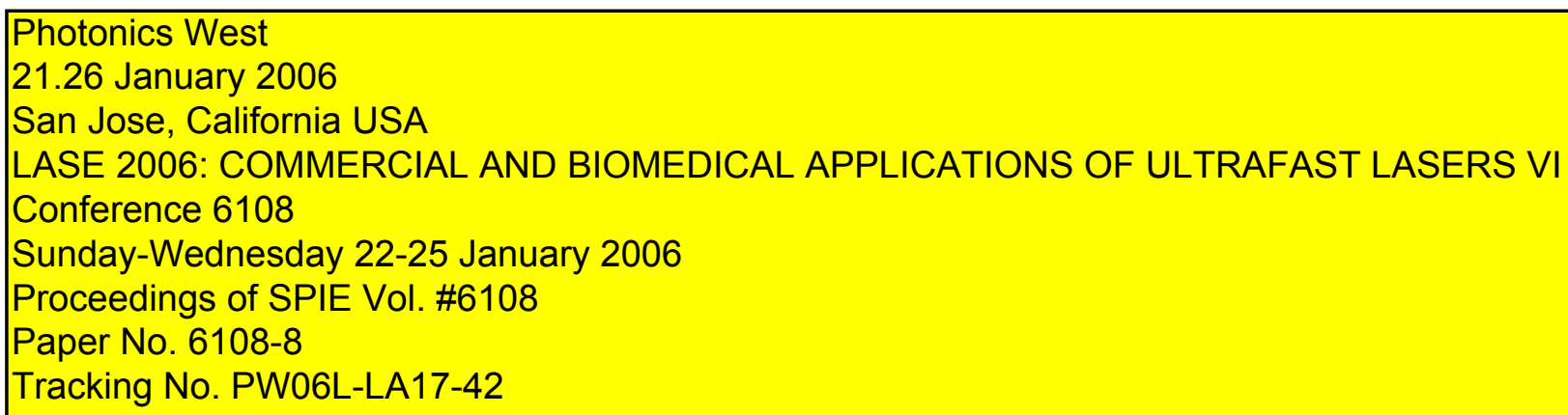




\section{Introduction}

Over the last two decades third harmonic generation (THG) has been widely used to identify two and three-photon resonances in solids and thin films. These measurements have served as a practical guide to the development of new optical materials and have informed theoretical understandings of the relationship between the electronic structure and optical properties of materials $[1,2]$.

Understanding the nonlinear optical properties of solution-phase materials, especially for biological molecules undergoing irradiation by ultra-fast pulsed lasers, has attained new relevance following the application of nonlinear laser scanning microscopies to the visualization of biological samples [3]. A single beam nonlinear microscopy approach may collect two photon fluorescence, second harmonic generation, THG, or any combination thereof for imaging contrast, but it will also drive multi-photon absorption and THG in the sample under study. The multi-photon absorption features of the different components of the sample indicate likely pathways for photo-toxicity, phototherapy, and shadowing effects. Thus quantitative information on the nonlinear properties of biological samples is of general interest to nonlinear microscopy in biology even when the properties are not directly participating in the primary contrast mechanisms of the microscopy. Clearly, such information is of particular import to the understanding of imaging modalities, such as THG, where contrast is usually provided by the sample itself and not exogenous chromophores.

The primary contrast mechanism in THG microscopy is related to sub-focal volume inhomogeneities in the optical properties of a sample. Under tight focusing conditions, the extent of THG increases dramatically when the focus spans an interface between two optically different materials $[4,5]$. This allows imaging based on THG to resolve otherwise transparent interfaces and inhomogeneities within the resolution of the confocal parameter and without the use of dyes [6-10]. THG has been used to image mitochondria [11], red blood cells [12, 13], embryonic development [7, 14], neurons [10], plasma flows [15], muscle fibers [14], skin biopsy samples [16], and most recently large intracellular lipid vesicles[17].

From an imaging perspective THG also holds the promise of functional signals, i.e., changes in THG intensity may reflect changes in the molecular structure or composition of a sample. Evidence for this can be seen in the sensitivity of THG image contrast to the density of optical solids, the aggregation state of polymers [18-20], and the concentration of intracellular $\left[\mathrm{Ca}^{2+}\right]$ in cultured human glial cells. [21]. Schaller et al [13] qualitatively showed that the near field THG image of an oxygenated dried red blood cell was best near an excitation wavelength of $\lambda=1.26 \mu \mathrm{m}$, which corresponds to a three-photon resonance in hemoglobin, near the $420 \mathrm{~nm}$ Soret band.

Only through an understanding of the nonlinear spectral characteristics of the samples under study can microscopists fully realize the potential of nonlinear excitation in imaging, probing, and perturbing biological structure and function. This is particularly evident with THG microscopy where every image may carry information regarding the physical and chemical state of the sample. These applications have driven the development of a quantitative ultrafast THG microspectroscopy that is readily accessible to existing laser scanning microscopes. In this study we apply and further develop this approach and characterize the THG spectra of several solutions with $\sim 100 \mathrm{fs}$ long, $1 \mathrm{~nJ}$ energy, laser pulses in the $750-1000 \mathrm{~nm}$ wavelength range. Our samples include pure water and hemoglobin solutions at physiological concentrations and in different oxidation states. 


\section{Micro-spectroscopy Method and the Nonlinear Spectra of Water}

\subsection{Third Harmonic Generation Z-scan procedure and the Third Harmonic Ratio, $\mathbf{R}_{\mathrm{THG}}$.}

THG micro-spectroscopy has its roots in Tsang's 1995 discovery, that THG from a focussed laser is greatly increased by the presence of an interface [5], and has been further developed by various researchers [22-24]. The basic Z-scan procedure applied to a sample of water is diagramed in Figure 1A-C. Both the interface between the water and the glass capillary container (Fig.1A) and the interface between the glass container and the air (Fig.1B) are scanned transversely through the focus of the laser beam. We collect the THG light on the far side of the sample using bandpass and UG-11 glass filters and a PMT (Hamamatsu R6353). The THG collected as each interface is scanned through the focus is shown in Fig.1C. Plotting the THG power $P^{(3)}(3 \omega)$ as a function of incidence power $P(\omega)$ the well known THG scaling law:

$$
P(3 \omega) \sim P^{3}(\omega)
$$

can be observed (Fig.1D) and used to insure the signal is THG. Correcting for reflection $r(\omega), r(3 \omega)$, and absorption $a(\omega), a(3 \omega)$ of the transmitted fundamental and the third harmonic light respectively allows for the formation of $\mathrm{R}_{\mathrm{THG}}$, the ratio of the cubic fit parameters derived for each interface:

$$
\mathrm{R}_{\mathrm{THG}}(\omega) \equiv \frac{\alpha_{\text {solution/glass }}(\omega)}{\alpha_{\text {glass/air }}(\omega)} \frac{\left[1-r^{2}(\omega)_{\text {glass/solution }}\right]^{3}\left[1-a(\omega)_{\text {glass }}\right]^{3}}{\left[1-r^{2}(3 \omega)_{\text {glass/solution }}\right]\left[1-a(3 \omega)_{\text {glass }}\right]}
$$

This ratio facilitates a power independent measurement of the nonlinear susceptibility $\chi^{(3)}(3 \omega)$ to be made relative to a standard material, such as the $\mathrm{SiO}_{2}$ substrate of the glass container (see next section). The process not only simplifies the complications involved in making absolute measurements, and verifies the source of the signal, but also provides some control on systematic drifts in the laser parameters over time and wavelength (i.e. beam divergence, collection efficiency, sample orientation, etc). The third harmonic spectrum $\mathrm{R}_{\mathrm{THG}}$ of water is shown relative to $\mathrm{SiO}_{2}(\mathrm{Fig}$.1E).

Figure 1. Z-scan and the Third Harmonic Ratio, $\mathrm{R}_{\mathrm{THG}}$.

A

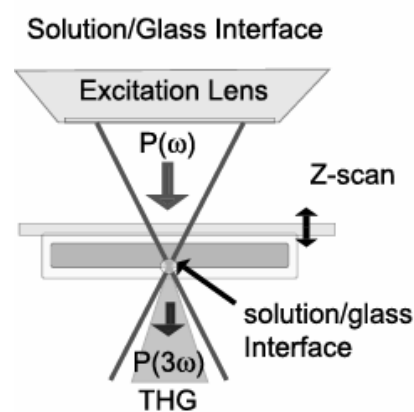

B

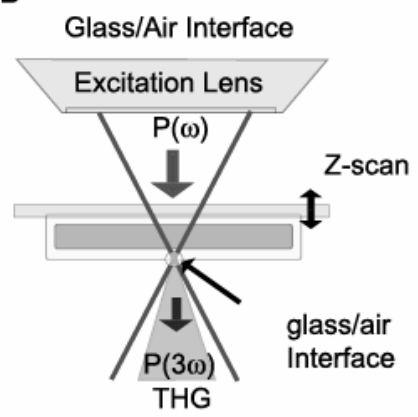

C

Third Harmonic Power, $\mathrm{P}(3 \omega)$ [arbs]

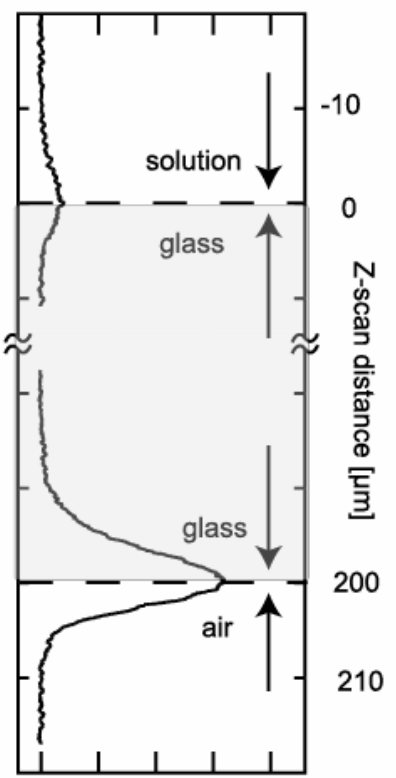

D

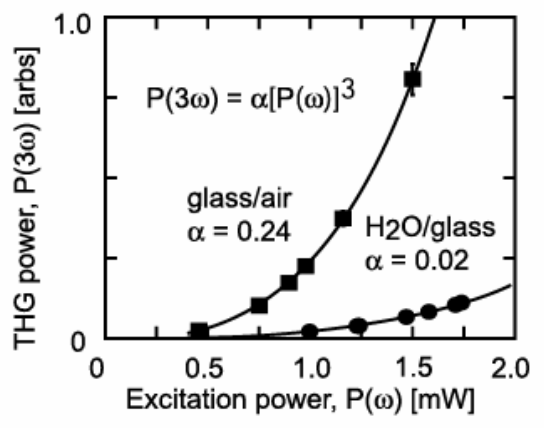

E

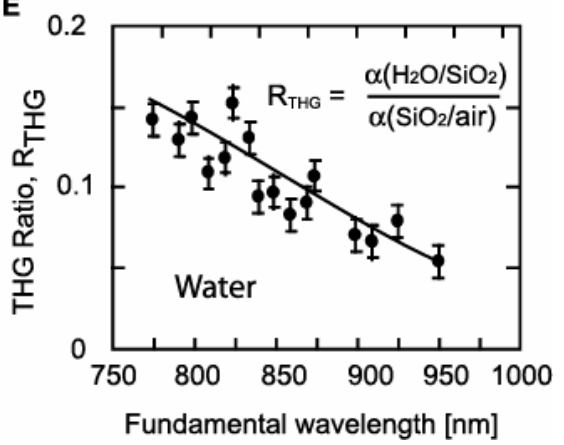




\subsection{Deriving $X^{(3)}(3 \omega)$ and one-photon resonance in the $X^{(3)}(3 \omega)$ spectra of water.}

Due to the possibility of destructive interference of the third harmonic wave generated on either side of the focus, the third harmonic ratio $\mathrm{R}_{\mathrm{THG}}$ may increase or decrease with resonance enhancement effects. For this reason, identifying multi-photon resonances in the nonlinear spectra of a sample requires the calculation of the nonlinear susceptibility $\mathrm{X}^{(3)}(3 \omega)$.

Figure 2. Deriving $X^{(3)}(3 \omega)[15,22,24-26]$ and one-photon resonance in the nonlinear spectra of water[1, 5, 23, 27].
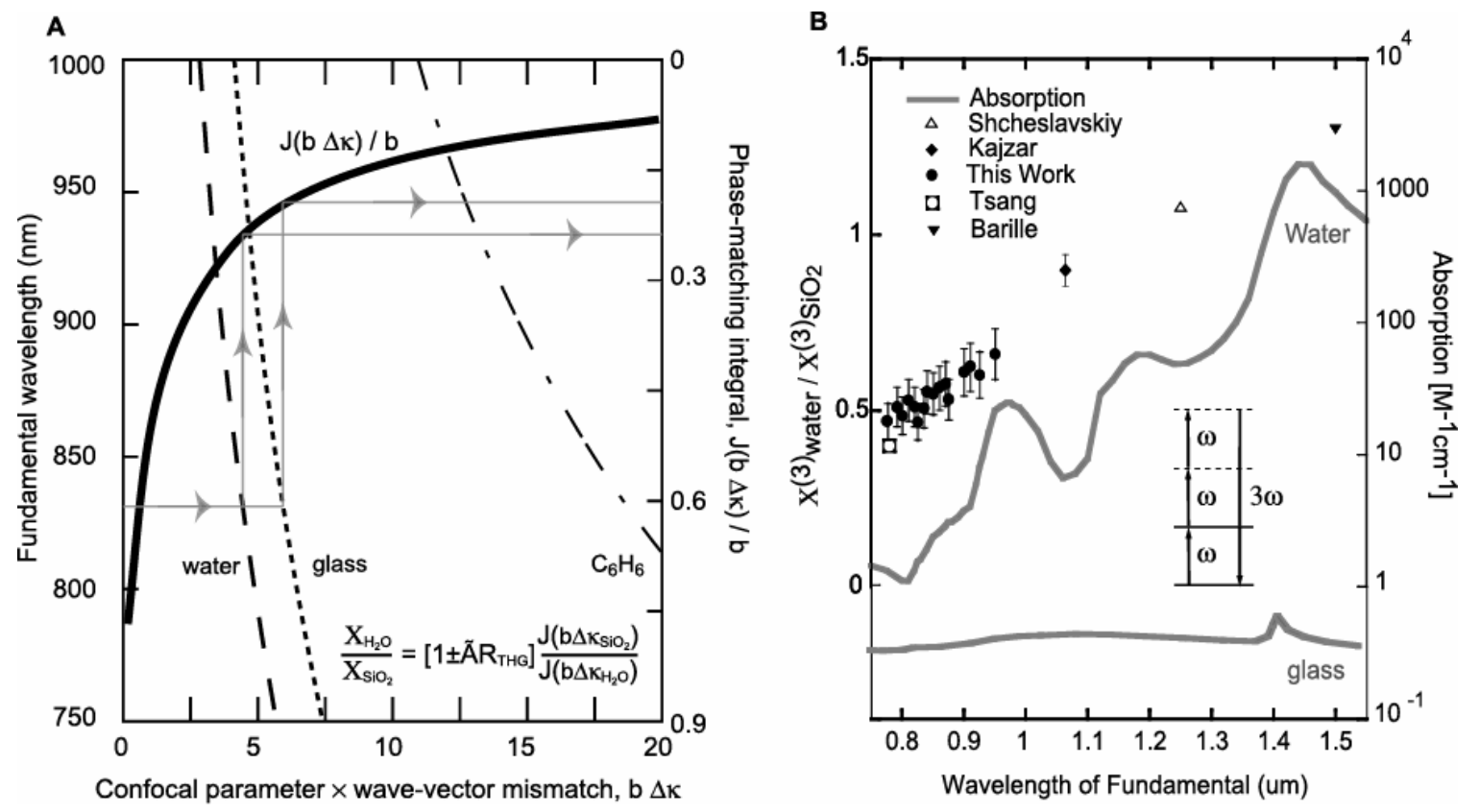

The ratio of the nonlinear susceptibilities is related to the third harmonic ratio $\mathrm{R}_{\mathrm{THG}}$ by $[22,24]$ :

$$
\frac{\chi_{\text {solution }}^{(3)}(\omega)}{\chi_{\text {glass }}^{(3)}(\omega)}=\frac{\mathrm{J}\left(\mathrm{b}_{\text {glass }} \Delta \kappa_{\text {glass }}\right) \mathrm{b}_{\text {glass }}(\omega)}{\mathrm{J}\left(\mathrm{b}_{\text {solution }} \Delta \kappa_{\text {solution }}\right) \mathrm{b}_{\text {solution }}(\omega)}\left\{\beta(\omega) \rho(\omega) \pm \sqrt{\mathrm{R}_{\mathrm{THG}}(\omega)}\right\}
$$

Where $\mathrm{b}$ is the confocal parameter (a measure of the axial extent of the focus), $\Delta \kappa$ is the wave-vector mismatch related to the phase matching characteristics of the fundamental and third harmonic wave in a given material:

$$
\Delta \kappa=6 \pi \frac{\mathrm{n}(\omega)-\mathrm{n}(3 \omega)}{\lambda}
$$

and $\mathrm{J}(\mathrm{b} \Delta \kappa)$ is a the dimensionless phase matching integral that can be numerically computed as a function of $\mathrm{b} \Delta \kappa$ (see Fig.2A) [15, 22]. The coefficient

$$
\beta(\omega) \equiv \frac{\mathrm{J}\left(\mathrm{b}_{\text {glass-solution }} \Delta \kappa_{\text {glass }}\right) \mathrm{b}_{\text {glass-solution }}(\omega)}{\mathrm{J}\left(\mathrm{b}_{\text {glass-air }} \Delta \kappa_{\text {glass }}\right) \mathrm{b}_{\text {glass-air }}(\omega)}
$$

accounts for possible changes in the confocal parameter of glass at the two interfaces due to aberrations and 


$$
\rho(\omega)=\sqrt{\frac{\left[1-r^{2}(\omega)_{\text {glasss solution }}\right]^{3}}{\left[1-r^{2}(3 \omega)_{\text {glasssolution }}\right]}}
$$

adjusts for reflections at the interfaces of the micro-slide capillary. The sign ambiguity in Eq. 3 must be resolved either by comparison with other measurements or through consideration of resonances [24]. The wave-vector mismatch $\Delta \kappa$ and thus the phase matching integral $\mathrm{J}(\mathrm{b} \Delta \kappa)$ require knowledge of a material's refractive index at both the fundamental and the third harmonic wavelength. Accurate models of the refractive index exist for water [25] and glass [26] and are used to calculate $\mathrm{b} \Delta \kappa$ (see the dashed curves in Figure 2A). The functional dependence of the phase matching integral for different materials at different wavelengths is illustrated in bold in Figure 2A [15, 22].

The $\mathrm{X}^{(3)}(3 \omega)$ values found for water here correspond well with literature measurements $[1,5,22,23]$ (see Figure $2 \mathrm{~B}$ ). The monotonic increase in the third order susceptiblity and the linear absorption [27] suggest that one-photon resonance dominates the $X^{(3)}(3 \omega)$ values of water in the near-infrared. Error bars are included when reported in the literature and suggest that the Z-scan THG approach is of comparable accuracy to other techniques.

\section{Nonlinear Spectra of Hemoglobin}

\subsection{Nonlinear spectra of Hemoglobin in different ligand binding states}

Solutions of hemoglobin (300881RO; Instrumentation Laboratories, Lexington, MA) at physiological concentrations $(\sim 2 \mathrm{mM})$ and in different ligand binding states have different nonlinear optical characteristics. THG power is shown to be reduced as deoxyhemoglobin becomes oxygenated at $921 \mathrm{~nm}$ (see Fig.3A, inset shows linear aborption). Differences in the nonlinear optical properties of hemoglobin can be seen across wavelengths and other ligand binding states. In Figure 3B the spectrally averaged third harmonic ratio for oxy, deoxy, and carboxy hemoglobin is shown along with the actual data for carboxyhemoglobin. Statistically significant differences between the susceptibility values of the different ligand states is reached at several wavelengths (see [24] for a detailed discussion of errors in this technique).

Figure 3. Nonlinear $\left(\mathrm{R}_{\mathrm{THG}}\right)$ spectra of hemoglobin in different ligand binding states

A THG intensity decreases with succesive oxygenation of deoxyhemoglobin

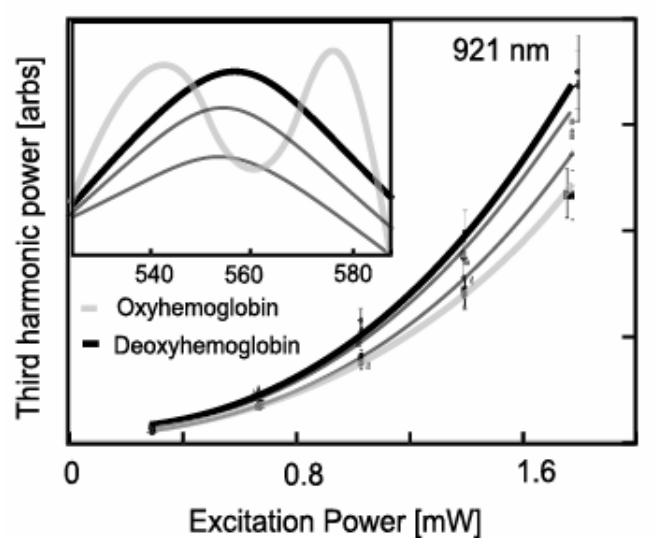

B

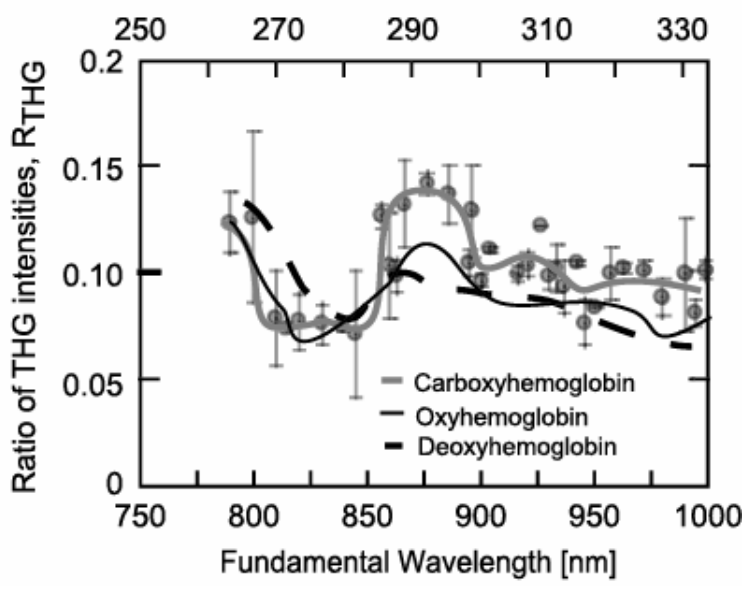




\subsection{Deriving $X^{(3)}(3 \omega)$ of a solute and two photon resonance in $X^{(3)}(3 \omega)$ the spectra of hemoglobin}

Extracting accurate values of $\mathrm{X}^{(3)}(3 \omega)$ for a solute from a solution requires that the sign of both the solvent and solute are disambiguated and that the volume fraction $v$ of the solute is determined. In an aqueous solution the relation

$$
\frac{\chi_{\text {solute }}^{(3)}(\omega)}{\chi_{\text {glass }}^{(3)}(\omega)}=\frac{\mathrm{J}\left(\mathrm{b}_{\text {glass }} \Delta \kappa_{\text {glass }}\right) \mathrm{b}_{\text {glass }}(\omega)}{\mathrm{J}\left(\mathrm{b}_{\text {solution }} \Delta \kappa_{\text {solution }}\right) \mathrm{b}_{\text {solution }}(\omega)}\left\{\beta(\omega) \rho(\omega)+\frac{1}{v}\left[ \pm \sqrt{\mathrm{R}_{\substack{\text { solution } \\ \text { sol }}}(\omega)}-(1-v) \sqrt{\mathrm{R}_{\substack{\text { THG } \\ \text { solvent }}}(\omega)}\right]\right\}
$$

holds [24], where all the terms are defined as before.

As in the case of a pure sample, the refractive index at both the fundamental and the third harmonic wavelength is required to calculate the phase matching integrals in Eq.7. Because refractive measurements in the UV are relatively rare it is likely that researchers will have to estimate these parameters. In order to approximate the index of refraction for hemoglobin (Fig.4B), we fit the linear absorption peaks of hemoglobin [28] (Fig.4A) with a series of Gaussian functions (see inset in Fig.4B) and employ a technique due to Latimer [29] based on a Taylor expansion of the KramersKronig relations [24]. For oxyhemoglobin this first order approximation fits within the errors of most of the concentration corrected measured values (Fig.4B) [30-32].

Figure 4. Linear absorption[28] and the refractive index of hemoglobin.
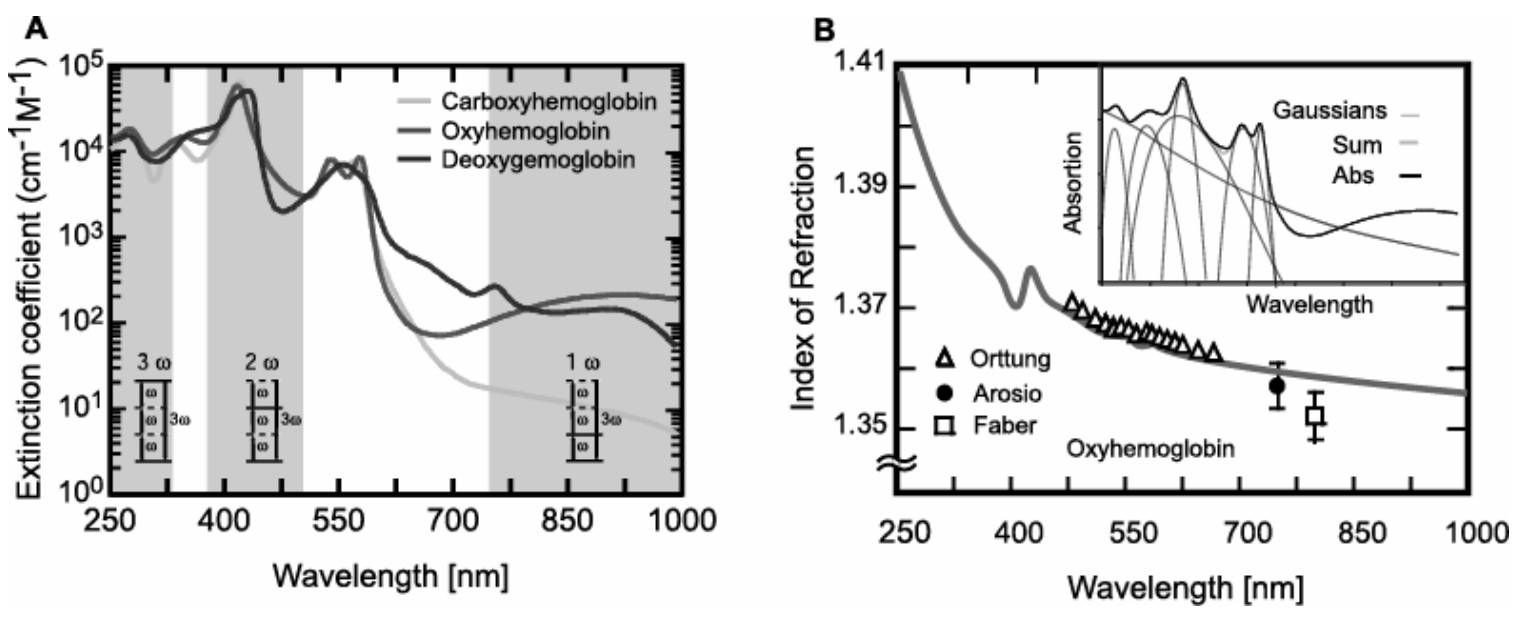

The fact that the third harmonic ratio $\mathrm{R}_{\mathrm{THG}}$, or equivalently image contrast in THG microscopy, may increase or decrease with resonance enhancement effects is related to the sign ambiguity in Equations $3 \& 7$. Thus, the identification of a multi-photon resonance in the nonlinear spectra of a sample can be used to help resolve the sign ambiguity in the nonlinear susceptibility calculation and vice a versa.

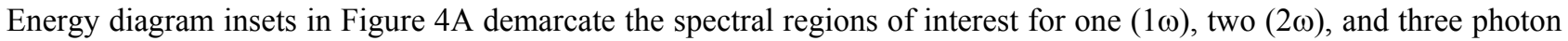
$(3 \omega)$ resonance in hemoglobin irradiated over the range of $770-1000 \mathrm{~nm}$ [28]. A comparison between these spectral regions and our derived values for the $\mathrm{X}^{(3)}(3 \omega)$ of hemoglobin shows dramatic evidence of two-photon resonance (Fig.5). The details involved in estimating the parameters necessary for the susceptibility calculation of hemoglobin are previously specified [24]. 
Figure 5. Two photon resonance in the $\mathrm{X}^{(3)}$ specta of oxy and deoxy-hemoglobin.

A

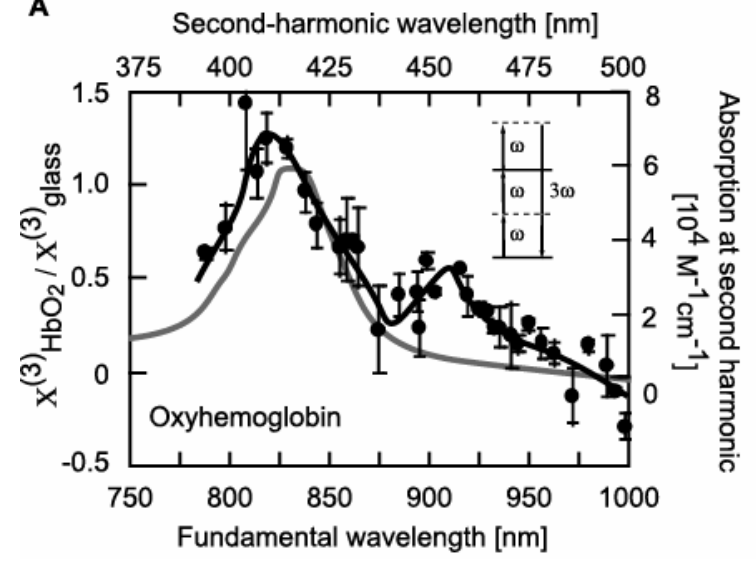

B Second-harmonic wavelength [nm]

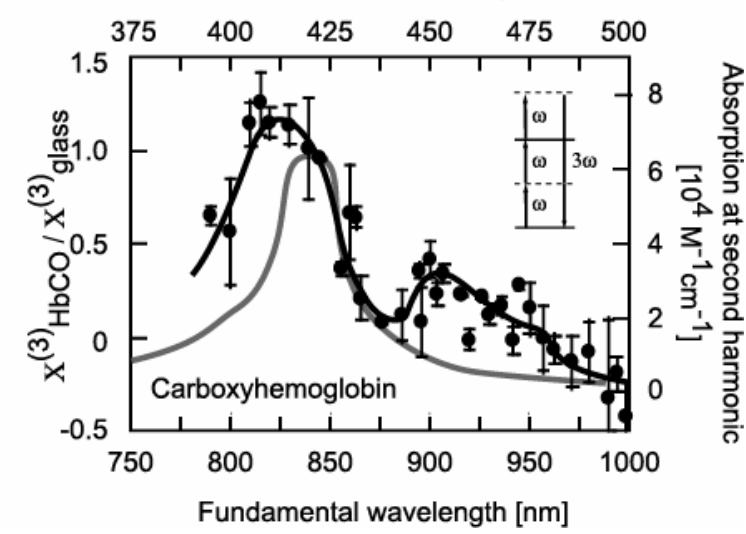

\section{Conclusion}

Most laser scanning microscopy rigs can be easily modified to accommodate the Z-scan based THG micro-spectroscopy approach outlined here. The technique does not require special sample cells and can be performed with the same laser energies and excitation objectives used in imaging. Careful accounting of reflection and transmission coefficients renders the measurements made with this technique as accurate as many made with much higher laser energies (see Fig.2B). This approach provides a new tool for laser scanning microscopists interested in identifying possible multiphoton toxicity, shadowing, or contrast mechanisms that may be latent in non-fluorescent or endogenous materials.

The third harmonic ratio $\mathrm{R}_{\mathrm{THG}}$, which corresponds to image contrast in a THG microscope, is shown to be a function of ligand binding state in hemoglobin. This finding should encourage nonlinear microscopists who are looking to use the nonlinear spectral properties of endogenous molecules as functional markers during in vivo nonlinear imaging.

We also demonstrate how the nonlinear susceptibility $\mathrm{X}^{(3)}(3 \omega)$ of a solute can be derived from the third harmonic ratio measurement from a solution with a known solvent. This allows us to easily identify a strong two photon resonance at $825 \mathrm{~nm}$ in physiological concentrations of oxy and carboxyhemoglobin solutions (Fig.5). These results provide guidance in the choice of excitation wavelength (e.g. for minimizing photodamage or maximizing shadowing effects) in the nonlinear imaging of blood.

\section{References}

1. Kajzar, F. and J. Messier, Third-harmonic generation in liquids. Physics Review. A, 1985. 32: p. 2352-2363.

2. Meredith, G.R., B. Buchalter, and C. Hanzlik, Third-order optical susceptibility determination by third harmonic generation. 2. Journal of Chemical Physics, 1983. 78: p. 1543-1551.

3. Denk, W., J.H. Strickler, and W.W. Webb, Two-photon laser scanning fluorescence microscopy. Science, 1990. 248: p. 73-76.

4. Barad, Y., et al., Nonlinear scanning laser microscopy by third harmonic generation. Applied Physics Letters, 1997. 70: p. 922-924.

5. $\quad$ Tsang, T.Y.F., Optical third harmonic generation at interfaces. Physical Review A, 1995. 52: p. 4116-4125. 
6. Reintjes, J., Nonlinear Optical Parametric Processes in Liquids and Gases. 1984, Washington, DC: Academic Press, INC.

7. Chu, S.-W., et al., In vivo developmental biology study using noninvasive multi-harmonic generation microscopy. Optics Express, 2003. 11: p. 3093-3099.

8. Muller, M., et al., 3D-microscopy of transparent objects using third harmonic generation. Journal of Microscopy, 1998. 191: p. 266-274.

9. $\quad$ Squier, J., et al., Third harmonic generation microscopy. Optics Express, 1998. 3: p. 315-321.

10. Yelin, D. and Y. Silberberg, Laser scanning third-harmonic microscopy in biology. Optics Express, 1999. 5: p. 169-175.

11. Barzda, V., et al. Second-and third-harmonic generation and multiphoton excitation fluorescence microscopy fro simultaneous imaging of cardiomyocytes. in Commercial and Biomedical Applicatrions of Ultrafast Lasers IV. 2004: Proceedings of SPIE.

12. Millard, A.C., et al., Third-harmonic generation microscopy by use of a compact, femtosecond fiber laser source. Applied Optics, 1999. 38: p. 7393-7397.

13. Schaller, R.D., J.C. Johnson, and R.J. Saykally, Nonlinear chemical imaging microscopy: Near-field third harmonic generation imaging of human blood cells. Analytical Chemistry, 2000. 72: p. 5361-5364.

14. Debarre, D., et al., Velocimetric third-harmonic generation microscopy: Micrometer-scale quantification of morphogenetic movements in unstained embryos. Optics Letters, 2004. 29: p. 2881-2883.

15. Naumov, A.N., et al., Third-harmonic generation in focused beams as a method of 3D microscopy of a laserproduced plasma. Optics Spectroscopy, 2001. 90: p. 778-783.

16. Sun, C.-K., et al., Multiharmonic-generation biopsy of skin. Optics Letters, 2003. 28(24): p. $2488-2490$.

17. Debarre, D., et al., Imaging lipid bodies in cells and tissues using third-harmonic generation microscopy. Nature Methods, 2006. 3: p. 47-53.

18. Hasegawa, T., et al., Excitonic resonant effect in the third-order nonlinear optical properties of blue-and red form polydiacetylene films. Chemical Physics Letters, 1990. 171: p. 239-244.

19. Squier, J.A. and M. Muller, Third-harmonic generation imaging of laser-induced breakdown in glass. Applied Optics, 1999. 38: p. 57895794.

20. Schaller, R.D., et al., Nanoscopic interchain aggreagate domain formation in conjugated polymer films studied by third harmoinic generation near-field scanning optical microscopy. Journal of Chemical Physics, 2002. 117: p. 6688-6698.

21. Canioni, L., et al., Imaging of Ca2+ intracellular dynamics with a third-harmonic generation microscope. Optics Letters, 2001. 26: p. 515-517.

22. Barille, R., et al., Nonlinearity measurements of thin films by third-harmonic-generation microscopy. Physical Review E, 2002. 66: p. 1-4.

23. Shcheslavskiy, V., G. Petrov, and V.V. Yakovlev, Nonlinear optical susceptibility measurements of solutions using third-harmonic generation on the interface. Applied Physics Letters, 2003. 82(22): p. 3982-3984.

24. Clay, G.O., et al., Spectroscopy of third harmonic generation: Evidence for resonances in model compounds and ligated hemoglobin. Journal of the Optical Society of America B, 2006: p. In Press.

25. Huibers, P.D.T., Models for the wavelength dependence of the index of refraction of water. Applied Optics, 1997. 36: p. 3785-3787.

26. Malitson, I.H., Interspecimen comparison of refractive index of fused silica. Journal of the Optical Society of America, 1965. 55: p. 1205-1209.

27. Segelstein, D.J., The complex refractive index of water, in physics. 1981, University of Missouri: Kansas City.

28. Lemberg, P. and J.W. Legge, Hematin Compounds and Bile Pigments. 1949, New York: Interscience Publishers, Inc.

29. Latimer, P., Anomalous Dispersion of CS2 and ChCl3: Theoretical predictions. Journal of the Optical Society of America, 1961. 51: p. 116-118.

30. Orttung, W. and J. Warner, Refractive index dispersion in equine hemoglobin solutions. The Journal of Physical Chemistry, 1965. 69: p. 3188-3190.

31. Faber, D., et al. Oxygen saturation dependent index of refraction of hemoglobin solutions assessed by OCT. in Proceedings of the SPIE. 2003.

32. Arosio, D., et al., Static and dynamic light scattering approach to the hydration of hemoglobin and its supertetramers in the presence of osmolites. Biopolymers, 2002. 63: p. 1-11. 